\title{
Impact of Sowing Period and Variety on Pod Borer, Helicoverpa armigera Hubner in Pigeonpea
}

\author{
G. D. Hadiya ${ }^{1 *}$, S.D. Patel $^{2}$, R. L. Chavadhari ${ }^{1}$, R. G. Machhar ${ }^{1}$ and C. B. Damor ${ }^{1}$ \\ ${ }^{1}$ Agricultural Research Station, Anand Agricultural University, Derol- 389 320, Dist. \\ Panchmahal, Gujarat, India \\ ${ }^{2}$ Directorate of Extension Education, Anand Agricultural University, Anand, Gujarat, India \\ *Corresponding author
}

A B S T R A C T

\begin{tabular}{|c|c|}
\hline $\begin{array}{l}\text { Ke y w o r d s } \\
\text { Helicoverpa } \\
\text { armigera, Variety, } \\
\text { Pigeonpea, sowing } \\
\text { period }\end{array}$ & $\begin{array}{l}\text { A field experiment was conducted to study the impact of sowing period }\left[24^{\text {th }}, 26^{\text {th }} \text {, }\right. \\
28^{\text {th }}, 31^{\text {st }} \text { and } 33^{\text {rd }} \text { Standard Meteorological Week (SMW)] and variety (AGT-2, } \\
\text { BDN-2 and Vaishali) on pod borer, Helicoverpa armigera in pigeonpea at } \\
\text { Agricultural Research Station, Anand Agricultural University, Derol, Dist. } \\
\text { Panchmahal, Gujarat, India during Kharif, 2014-15, 2015-16, 2017-18 and 2018- }\end{array}$ \\
\hline Article Info & $\begin{array}{l}\text { 19. Results of the study revealed that late sown }\left(31^{\text {st }} \text { and } 33^{\text {ta }} \text { SMW }\right) \\
\text { pigeonpeacrop had significantly lower population of } H \text {. armigeraand significantly }\end{array}$ \\
\hline $\begin{array}{l}\text { Accepted: } \\
\text { 17 June } 2020 \\
\text { Available Online: } \\
10 \text { July } 2020\end{array}$ & $\begin{array}{l}\text { lower per cent pod damage recorded at green pod as well as harvest stage. The } \\
\text { effect of variety on per cent pod damage at green pod and harvest stage was non- } \\
\text { significant. Grain yield was also not affected significantly by sowing period and } \\
\text { variety. }\end{array}$ \\
\hline
\end{tabular}

\section{Introduction}

Pigeonpea [Cajanus cajan (L.) Millsp.], commonly known as redgram, Tur, Arhar etc., is an erect and short-lived perennial shrub legume (Sharma et al., 2010). India is the world's largest producer and consumer of pulses including pigeonpea. India accounts for about $75 \%$ of world production (Sharma et al., 2010). In India pigeonpea is cultivated in area of 44.38 lakh hectare, while its production is 42.89 lakh tonne and productivity is $967 \mathrm{~kg} /$ hectare (Anonymous, 2017-18). Maharashtra, Uttar Pradesh, Madhya Pradesh, Karnataka, Gujarat, Andhra
Pradesh, Tamilnadu and Bihar are the major pigeonpea growing states. The area under pigeonpea cultivation in Gujarat is 2.71 lakh hectare, whereas its production is 3.37 lakh tonne with productivity of $1243 \mathrm{~kg} /$ hectare (Anonymous, 2017-18). More than 90 insect species were reported to feed on pigeonpea crop in India (Davis and Lateef, 1975). Amongst them, some are important and a few are the considered as "key pest". The important pests of pigeonpea in central zone of India are Helicoverpa armigera, Maruca vitrata, Melangromy zaobtusa, Lampides boeticus and Clavigralla gibbosa. Among these H.armigera, M. obtusa and M. Vitrata 
are key pests. Pod borer, H.armigera is the most dreaded and polyphagous pest of pigeonpea worldwide (Patel, 2019). Its preference for flowering and fruiting parts results in heavy loss up to $60 \%$ or more under subsistence agriculture in the tropics. The annual monetary losses were estimated at US $\$ 400$ in pigeonpea per hectare(Anonymous, 2007).

Farmers rely heavily on chemical insecticides to manage insect pest. Chemical insecticides cause many side effects. To overcome the problem of the indiscriminate use of pesticides, eco-friendly techniques such as agronomic practices can be utilized. One such method is altering the sowing dates in order to escape the peak activity of the insect by the crop. Date of sowing has a great impact on the incidence of the pest which may be attributed to the difference in weather condition (Deka et al., 1989, Yadava et al., 1991, Cumming, 2011). Hence, it is essential to find out optimum sowing periodwhich help the crop to escape damage of insect pests. Certain varieties are less damaged by insect pests. Therefore, the present study was conducted to study the impact of sowing period and variety on pod borer, H.armigera in pigeonpea.

\section{Materials and Methods}

The study on impact of sowing period and variety on pod borer, $H$. armigera in pigeonpea was carried out at Agricultural Research Station, Anand Agricultural University, Derol, Dist. Panchmahal, Gujarat, India during Kharif, 2014-15, 2015-16, $2017-$ 18 and 2018-19. The experiment was laid out in split plot design with three replications; sowing period was taken as main plot treatment, whereas variety was taken as sub plot treatments. There were five sowing periods viz., $24^{\text {th }}, 26^{\text {th }}, 28^{\text {th }}, 31^{\text {st }}$ and $33^{\text {rd }}$ Standard Meteorological Week (SMW).
Three varieties viz., BDN-2, AGT-2 and Vaishali were evaluated as sub plot treatment. Pigeonpea crop was sown as per the decided period of sowing and variety with a spacing $90 \times 20 \mathrm{~cm}$. The gross plot size was $4.5 \times 5 \mathrm{~m}$, whereas net plot size was 2.7 x $4.6 \mathrm{~m}$. All agronomic practices were followed to raise the crop. All the plots were kept free from application of any insecticide.

For recording the observations of $H$. armigera, 5 plants were selected randomly from each net plot area and number of larvae of $H$. armigera was counted from whole plant at weekly interval starting from bud formation stage to harvest. In order to record the pod damage at green pod stage and at harvest stage, 100 pods were randomly plucked from each net plot area and pods were segregated into healthy and damaged. Based on this per cent pod damage was calculated. At harvest, grain yield was record from each net plot and it was converted into $\mathrm{kg} / \mathrm{ha}$. The data on larval population of $H$. armigera, per cent pod damage and grain yield were subjected to ANOVA to draw the conclusion.

\section{Results and Discussion}

\section{Impact of sowing period and varietyon larval population of $\boldsymbol{H}$. armigera}

\section{Impact of sowing period}

The data on the impact of sowing period on larval population of $H$. armigera are given in Table 1. Results show that during the year 2014-15, sowing period significantly affected the larval population of $H$. armigera. Significantly lowest larval population was recorded in $33^{\text {rd }}$ SMW (0.48 larva/plant) and significantly highest population was recorded in $24^{\text {th }}$ SMW (1.79 larvae/plant).

In the year 2015-16, larval population of $H$. armigera was significantly affected by 
sowing period. Significantly lowest larval population was recorded in $31^{\text {st }}$ SMW (0.19 larva/plant) and highest population was recorded in $24^{\text {th }}$ SMW (0.88 larva/plant).

During the year 2016-17, significantly lowest larval population was observed in the crop sown in $31^{\text {st }}$ SMW (0.39 larva/plant) and it was at par with $33^{\text {rd }}$ SMW (0.51 larva/plant). During the year 2017-18, sowing period had no significant influence on larval population of H. armigera.

Pooled analysis of four years' data indicated that sowing period significantly affected larval population of pod borer, $H$. armigera in pigeonpea. Overall, there was significant decrease in larval population with delay in sowing period from $28^{\text {th }}$ SMW (1.06 larva/plant) to $33^{\text {rd }}$ SMW (0.43 larva/plant). Significantly lowest larval population was observed in $33^{\text {rd }}$ SMW and it was at par with $31^{\text {st }}$ SMW (0.46 larva/plant).

Patelet al., (2019) while studying the effect of sowing period and variety on pod borer, $H$. armigera recorded the significantly lowest larval population (1.86 larvae/plant) in pigeonpea crop sown in $33^{\text {rd }}$ SMW as compared to early sowing ( $\left.24^{\text {th }} \mathrm{SMW}\right)$.

\section{Impact of variety}

The data on the impact of variety on larval population of $H$. armigeraare given in Table 1. Results show that during the year 201415 significantly lowest larval population was recorded in AGT-2 (1.06 larvae/plant) and it was at par with BDN-2 (1.09 larvae/plant). Significantly highest population of $H$. armigera was recorded in Vaishali (1.34 larvae/plant). Almost similar trend was observed during the year 2015-16.

In the year 2016-17, BDN-2 (0.54 larva/plant) recorded significantly lowest larval population as compared to AGT-2 (0.72 larva/plant) and BDN-2 (0.75 larva/plant). During the year 2017-18, larval population $H$. armigera was not significantly affected by variety. Pooled analysis also showed nonsignificant effect of variety on larval population $H$. armigera.

Impact of sowing period and variety on pod damage

\section{Impact of sowing period and variety on pod damage at green pod stage}

\section{Impact of sowing period}

The data on per cent pod damage by $H$. armigera recorded at the time of the green pod stage of pigeonpea crop are presented in Table 2. During year 2014-15, significantly lowest pod damage $(2.56 \%)$ was observed at $33^{\text {rd }}$ SMW and it was at par with $31^{\text {st }}$ SMW $(2.76 \%)$. Significantly highest pod damage $(5.25 \%)$ was recorded in $24^{\text {th }}$ SMW.

In the year 2015-16, significantly lowest per cent pod damage $(4.26 \%)$ was recorded in $33^{\text {rd }}$ SMW and it was at par with $31^{\text {st }}$ SMW $(4.31 \%)$ and $26^{\text {th }}$ SMW (5.32\%). Significantly highest grain damage was recorded in $24^{\text {th }}$ SMW (6.62\%).

During the year 2016-17, significantly lowest per cent pod damage was observed in crop sown on $31^{\text {st }}$ SMW (6.34\%) and it was at par with $24^{\text {th }}$ SMW $(6.88 \%)$. Significantly highest pod damage was recorded when crop sown in $26^{\text {th }}$ SMW (13.78\%).

In the year 2017-18, the per cent pod damage varied from 3.09 to 6.18 per cent. Significantly lowest per cent pod damage was observed in crop sown on $33^{\text {rd }}$ SMW $(3.09 \%)$ and highest per cent pod damage was recorded when crop sown in $26^{\text {th }}$ SMW $(6.18 \%)$. 
Table.1 Impact of sowing period and variety on larval population of H. armigera in pigeonpea

\begin{tabular}{|c|c|c|c|c|c|}
\hline \multirow[t]{2}{*}{ Treatment } & \multicolumn{5}{|c|}{ No. of larvae / plant } \\
\hline & 2014-15 & 2015-16 & 2016-17 & 2017-18 & Pooled \\
\hline \multicolumn{6}{|l|}{ Sowing Period (Main Plot Treatment) } \\
\hline \multirow[t]{2}{*}{$24^{\text {th }}$ SMW ( $2^{\text {nd }}$ week of June) } & $1.51^{\mathrm{e}}$ & $1.18^{\mathrm{e}}$ & $1.18^{\mathrm{d}}$ & $1.12^{\mathrm{a}}$ & $1.25^{\mathrm{b}}$ \\
\hline & (1.79) & $(0.88)$ & $(0.89)$ & $(0.76)$ & (1.06) \\
\hline \multirow[t]{2}{*}{$26^{\text {th }}$ SMW ( $4^{\text {th }}$ week of June) } & $1.47^{\mathrm{d}}$ & $1.08^{\mathrm{d}}$ & $1.17^{\mathrm{d}}$ & $1.07^{\mathrm{a}}$ & $1.20^{\mathrm{b}}$ \\
\hline & (1.66) & $(0.67)$ & $(0.86)$ & $(0.65)$ & $(0.93)$ \\
\hline \multirow{2}{*}{$28^{\text {th }}$ SMW ( $2^{\text {nd }}$ week of July) } & $1.36^{\mathrm{c}}$ & $0.95^{\mathrm{c}}$ & $1.11^{\mathrm{c}}$ & $1.10^{\mathrm{a}}$ & $1.13^{\mathrm{b}}$ \\
\hline & $(1.34)$ & $(0.40)$ & $(0.72)$ & $(0.71)$ & $(0.77)$ \\
\hline \multirow[t]{2}{*}{$31^{\text {st }}$ SMW ( $4^{\text {th }}$ week of July) } & $1.12^{\mathrm{b}}$ & $0.55^{\mathrm{a}}$ & $0.94^{\mathrm{a}}$ & $1.01^{\mathrm{a}}$ & $0.98^{\mathrm{a}}$ \\
\hline & $(0.75)$ & $(0.19)$ & $(0.39)$ & $(0.53)$ & $(0.46)$ \\
\hline \multirow[t]{2}{*}{$3^{\text {rd }}$ SMW ( $2^{\text {nd }}$ week of August) } & $0.99^{\mathrm{a}}$ & $0.85^{\mathrm{b}}$ & $1.00^{\mathrm{b}}$ & $1.02^{\mathrm{a}}$ & $0.96^{\mathrm{a}}$ \\
\hline & $(0.48)$ & $(0.22)$ & $(0.51)$ & $(0.54)$ & $(0.43)$ \\
\hline S. Em. \pm D & 0.01 & 0.02 & 0.01 & 0.004 & 0.042 \\
\hline $\mathbf{Y}$ & -- & -- & -- & -- & 0.009 \\
\hline D X Y Y & -- & -- & -- & -- & 0.019 \\
\hline C. D. at $5 \%$ & 0.04 & 0.06 & 0.05 & NS & 0.128 \\
\hline $\mathbf{Y}$ & -- & -- & -- & -- & 0.025 \\
\hline D X Y Y & -- & -- & -- & -- & 0.056 \\
\hline C.V. (\%) & 6.13 & 13.41 & 9.06 & 17.60 & 11.73 \\
\hline \multicolumn{6}{|l|}{ Variety (Sub Plot Treatment) } \\
\hline \multirow[t]{2}{*}{ BDN-2 } & $1.26^{\mathrm{a}}$ & $1.01^{\mathrm{b}}$ & $1.02^{\mathrm{a}}$ & $1.09^{\mathrm{a}}$ & $1.09^{\mathrm{a}}$ \\
\hline & (1.09) & $(0.51)$ & $(0.54)$ & $(0.70)$ & $(0.70)$ \\
\hline \multirow{2}{*}{ AGT-2 } & $1.25^{\mathrm{a}}$ & $0.93^{\mathrm{a}}$ & $1.10^{\mathrm{b}}$ & $1.04^{\mathrm{a}}$ & $1.08^{\mathrm{a}}$ \\
\hline & (1.06) & $(0.36)$ & $(0.72)$ & $(0.58)$ & $(0.66)$ \\
\hline \multirow{2}{*}{ Vaishali } & $1.36^{\mathrm{b}}$ & $1.01^{\mathrm{b}}$ & $1.12^{\mathrm{b}}$ & $1.06^{\mathrm{a}}$ & $1.14^{\mathrm{a}}$ \\
\hline & (1.34) & $(0.52)$ & $(0.75)$ & $(0.63)$ & $(0.79)$ \\
\hline S. Em. $\pm V$ & 0.02 & 0.02 & 0.01 & 0.002 & 0.02 \\
\hline $\mathbf{P}$ & 0.02 & 0.02 & 0.02 & 0.004 & 0.00 \\
\hline D x V & 0.04 & 0.03 & 0.03 & 0.011 & 0.03 \\
\hline V X Y & -- & -- & -- & -- & 0.017 \\
\hline D XP & 0.04 & 0.03 & 0.03 & 0.018 & 0.08 \\
\hline$V \times P$ & 0.05 & 0.04 & 0.04 & 0.011 & 0.03 \\
\hline$Y \times D \times V$ & -- & -- & -- & -- & 0.04 \\
\hline$Y \times D \times P$ & -- & -- & -- & -- & 0.05 \\
\hline$Y \times V \times P$ & -- & -- & -- & -- & 0.04 \\
\hline$D \times V \times P$ & 0.08 & 0.08 & 0.07 & 0.053 & 0.06 \\
\hline$Y \times D \times V \times P$ & -- & -- & -- & -- & 0.09 \\
\hline C. D. at 5\% & 0.05 & 0.04 & 0.04 & NS & NS \\
\hline $\mathbf{P}$ & 0.06 & 0.05 & 0.05 & 0.01 & 0.02 \\
\hline D x V & 0.10 & 0.09 & 0.09 & 0.03 & NS \\
\hline V X Y & -- & -- & -- & -- & 0.047 \\
\hline D x P & 0.10 & 0.09 & 0.09 & 0.05 & NS \\
\hline $\mathbf{V} \times P$ & NS & 0.12 & NS & 0.03 & NS \\
\hline$Y \times D \times V$ & -- & -- & -- & -- & 0.11 \\
\hline$Y \times D \times P$ & -- & -- & -- & -- & 0.14 \\
\hline$Y \times V \times P$ & -- & -- & -- & -- & 0.11 \\
\hline$D \times V \times P$ & 0.23 & 0.21 & NS & 0.15 & 0.05 \\
\hline$Y \times D \times V \times P$ & -- & -- & -- & -- & 0.24 \\
\hline C.V. (\%) & 11.29 & 13.26 & 11.99 & 14.80 & 13.34 \\
\hline
\end{tabular}

Note: Figures outside parenthesis are $\sqrt{x+0.5}$ transformed value and those inside parenthesis are retransformed values, Treatment means with the letter(s) in common are at par by DNMRT at 5\% level of significance. NS: Non-Significant, SMW: Standard Meteorological Week 
Table.2 Impact of sowing period and variety on pod damage at green pod stage due to $H$. armigera in pigeonpea

\begin{tabular}{|c|c|c|c|c|c|}
\hline \multirow[t]{2}{*}{ Treatment } & \multicolumn{5}{|c|}{ Pod damage (\%) } \\
\hline & 2014-15 & 2015-16 & 2016-17 & 2017-18 & Pooled \\
\hline \multicolumn{6}{|l|}{ Sowing Period (Main Plot Treatment) } \\
\hline \multirow[t]{2}{*}{$24^{\text {th }}$ SMW ( $2^{\text {nd }}$ week of June $)$} & $13.25^{\mathrm{c}}$ & $14.91^{\mathrm{c}}$ & $15.21^{\mathrm{ab}}$ & $14.16^{\mathrm{c}}$ & $14.14^{\mathrm{ab}}$ \\
\hline & $(5.25)$ & $(6.62)$ & $(6.88)$ & $(5.98)$ & $(5.96)$ \\
\hline \multirow[t]{2}{*}{$26^{\text {th }}$ SMW ( $4^{\text {th }}$ week of June) } & $12.34^{\mathrm{b}}$ & $13.34^{\mathrm{ab}}$ & $21.79^{c}$ & $14.39^{\mathrm{d}}$ & $15.25^{\mathrm{b}}$ \\
\hline & $(4.57)$ & $(5.32)$ & $(13.78)$ & $(6.18)$ & $(6.92)$ \\
\hline \multirow{2}{*}{$28^{\text {th }}$ SMW ( $2^{\text {nd }}$ week of July) } & $11.63^{\mathrm{b}}$ & $14.59^{\mathrm{bc}}$ & $17.10^{\mathrm{b}}$ & $14.29^{\text {cd }}$ & $14.36^{\mathrm{ab}}$ \\
\hline & $(4.06)$ & $(6.35)$ & $(8.65)$ & $(6.09)$ & $(6.15)$ \\
\hline \multirow[t]{2}{*}{$31^{\text {st }}$ SMW $\left(4^{\text {th }}\right.$ week of July $)$} & $9.57^{\mathrm{a}}$ & $11.98^{\mathrm{a}}$ & $14.58^{\mathrm{a}}$ & $12.64^{\mathrm{b}}$ & $12.18^{\mathrm{a}}$ \\
\hline & $(2.76)$ & $(4.31)$ & $(6.34)$ & $(4.79)$ & $(4.45)$ \\
\hline \multirow[t]{2}{*}{$33^{\text {rd }}$ SMW ( $2^{\text {nd }}$ week of August $)$} & $9.21^{\mathrm{a}}$ & $11.92^{\mathrm{a}}$ & $16.67^{\mathrm{b}}$ & $10.12^{\mathrm{a}}$ & $11.98^{\mathrm{a}}$ \\
\hline & (2.56) & $(4.26)$ & $(8.23)$ & (3.09) & $(4.31)$ \\
\hline S. Em. \pm & 0.27 & 0.50 & 0.64 & 0.06 & 0.778 \\
\hline $\mathbf{Y}$ & -- & -- & -- & -- & 0.345 \\
\hline D $\times \mathbf{Y}$ & -- & -- & -- & -- & 0.770 \\
\hline \multirow[t]{2}{*}{ C. D. at 5\% } & 0.83 & 1.57 & 1.99 & 0.19 & 2.396 \\
\hline & -- & -- & -- & -- & 0.99 \\
\hline D x Y & -- & -- & -- & -- & 2.220 \\
\hline C.V. $(\%)$ & 10.13 & 16.01 & 15.83 & 8.16 & 14.06 \\
\hline \multicolumn{6}{|l|}{ Variety (Sub Plot Treatment) } \\
\hline \multirow[t]{2}{*}{ BDN-2 } & $11.07^{\mathrm{a}}$ & $13.29^{\mathrm{b}}$ & $16.78^{\mathrm{a}}$ & $14.60^{c}$ & $13.88^{\mathrm{a}}$ \\
\hline & (3.69) & $(5.28)$ & (8.33) & (6.36) & $(5.75)$ \\
\hline \multirow{2}{*}{ AGT-2 } & $11.16^{\mathrm{a}}$ & $11.80^{\mathrm{a}}$ & $17.05^{\mathrm{a}}$ & $11.29^{\mathrm{a}}$ & $12.71^{\mathrm{a}}$ \\
\hline & $(3.75)$ & $(4.18)$ & $(8.60)$ & $(3.83)$ & $(4.84)$ \\
\hline \multirow[t]{2}{*}{ Vaishali } & $11.38^{\mathrm{a}}$ & $14.96^{\mathrm{c}}$ & $17.37^{\mathrm{a}}$ & $13.47^{b}$ & $14.15^{\mathrm{a}}$ \\
\hline & $(3.89)$ & $(6.66)$ & $(8.91)$ & $(5.43)$ & $(5.98)$ \\
\hline S. Em. \pm & 0.35 & 0.40 & 0.48 & 0.06 & 0.53 \\
\hline \multirow{2}{*}{$\begin{array}{r}\mathbf{P} \\
D \times V\end{array}$} & 0.28 & 0.32 & 0.39 & 0.04 & 1.46 \\
\hline & 0.49 & 0.56 & 0.68 & 0.29 & 1.17 \\
\hline V X Y & -- & -- & -- & -- & 0.50 \\
\hline D XP & 0.78 & 0.89 & 1.08 & 0.19 & 1.11 \\
\hline $\mathbf{V} \times P$ & 0.63 & 0.73 & 0.88 & 0.11 & 0.63 \\
\hline Y X D x V & -- & -- & -- & -- & 1.11 \\
\hline Y x D x P & -- & -- & -- & -- & 0.91 \\
\hline$Y \times V \times P$ & -- & -- & -- & -- & 0.71 \\
\hline $\mathbf{D} \times \mathbf{V} \times \mathbf{P}$ & 1.10 & 1.26 & 1.53 & 0.57 & 1.28 \\
\hline$Y \times D \times V \times P$ & -- & -- & -- & -- & 1.58 \\
\hline C. D. at 5\% & NS & 1.13 & NS & 0.16 & NS \\
\hline $\mathbf{P}$ & 0.81 & 0.92 & 1.12 & 0.11 & NS \\
\hline D x V & NS & 1.60 & 1.94 & 0.81 & NS \\
\hline V X Y & -- & -- & -- & -- & 1.38 \\
\hline $\mathbf{D} \times \mathbf{P}$ & NS & 2.52 & 3.07 & 0.54 & NS \\
\hline $\mathrm{V} \times P$ & NS & 2.06 & 2.50 & NS & NS \\
\hline Y x D x V & -- & -- & -- & -- & 3.09 \\
\hline$Y \times D \times P$ & -- & -- & -- & -- & 2.52 \\
\hline $\mathbf{Y} \times \mathbf{V} \times \mathbf{P}$ & -- & -- & -- & -- & 1.95 \\
\hline$D \times V \times P$ & NS & NS & 4.33 & NS & NS \\
\hline$Y \times D \times V \times P$ & -- & -- & -- & -- & 4.37 \\
\hline C.V. (\%) & 16.98 & 16.30 & 15.47 & 13.05 & 17.59 \\
\hline
\end{tabular}

Note: Figures outside parenthesis are arcsine transformed value and those inside parenthesis are retransformed values, Treatment means with the letter(s) in common are at par by DNMRT at 5\% level of significance. NS: Non-Significant, SMW: Standard Meteorological Week 
Table.3 Impact of sowing period and variety on pod damage at harvest due to $H$. armigerain pigeonpea

\begin{tabular}{|c|c|c|c|c|c|}
\hline \multirow[t]{2}{*}{ Treatment } & \multicolumn{5}{|c|}{ Pod damage (\%) } \\
\hline & 2014-15 & 2015-16 & 2016-17 & 2017-18 & Pooled \\
\hline \multicolumn{6}{|c|}{ Sowing Period (Main Plot Treatment) } \\
\hline \multirow[t]{2}{*}{$24^{\text {th }}$ SMW ( $2^{\text {nd }}$ week of June $)$} & $18.54^{\mathrm{c}}$ & $21.56^{\mathrm{c}}$ & $17.82^{\mathrm{bc}}$ & $13.79^{\mathrm{d}}$ & $17.93^{\mathrm{d}}$ \\
\hline & $(10.11)$ & $(13.50)$ & $(9.37)$ & $(5.68)$ & $(9.48)$ \\
\hline \multirow[t]{2}{*}{$26^{\text {th }}$ SMW ( $4^{\text {th }}$ week of June) } & $19.00^{c}$ & $18.45^{\mathrm{b}}$ & $19.55^{\mathrm{c}}$ & $12.45^{\mathrm{bc}}$ & $17.37^{\mathrm{cd}}$ \\
\hline & $(10.60)$ & $(10.02)$ & $(11.20)$ & $(4.65)$ & $(8.91)$ \\
\hline \multirow[t]{2}{*}{$28^{\text {th }}$ SMW ( $\left(2^{\text {nd }}\right.$ week of July $)$} & $15.17^{\mathrm{b}}$ & $16.85^{\mathrm{ab}}$ & $23.30^{\mathrm{d}}$ & $11.72^{\mathrm{ab}}$ & $16.76^{\mathrm{c}}$ \\
\hline & $(6.85)$ & $(8.40)$ & $(15.64)$ & $(4.13)$ & $(8.32)$ \\
\hline \multirow[t]{2}{*}{$31^{\text {st }}$ SMW ( $4^{\text {th }}$ week of July) } & $11.80^{\mathrm{a}}$ & $16.53^{\mathrm{a}}$ & $15.25^{\mathrm{ab}}$ & $12.81^{\mathrm{cd}}$ & $14.10^{\mathrm{ab}}$ \\
\hline & $(4.18)$ & $(8.10)$ & $(6.92)$ & $(4.92)$ & $(5.93)$ \\
\hline \multirow[t]{2}{*}{$3^{\text {rd }}$ SMW ( $2^{\text {nd }}$ week of August $)$} & $10.67^{\mathrm{a}}$ & $16.19^{\mathrm{a}}$ & $12.20^{\mathrm{a}}$ & $11.50^{\mathrm{a}}$ & $12.64^{\mathrm{a}}$ \\
\hline & $(3.43)$ & $(7.77)$ & $(4.47)$ & $(3.97)$ & $(4.79)$ \\
\hline S. Em. \pm & 0.518 & 0.665 & 1.014 & 0.30 & 1.19 \\
\hline $\mathbf{Y}$ & -- & -- & -- & -- & 0.30 \\
\hline $\mathbf{D} \times \mathbf{Y}$ & -- & -- & -- & -- & 0.68 \\
\hline C. D. at $5 \%$ & 1.79 & 2.30 & 3.508 & 0.97 & 3.68 \\
\hline $\mathbf{Y}$ & -- & -- & -- & -- & 0.87 \\
\hline $\mathbf{D} \times \mathbf{Y}$ & -- & -- & -- & -- & 1.95 \\
\hline C.V. $(\%)$ & 10.34 & 11.13 & 17.26 & 7.20 & 12.87 \\
\hline \multicolumn{6}{|l|}{ Variety (Sub Plot Treatment) } \\
\hline \multirow[t]{2}{*}{ BDN-2 } & $14.91^{\mathrm{a}}$ & $16.97^{\mathrm{a}}$ & $15.95^{\mathrm{a}}$ & $10.72^{\mathrm{a}}$ & $14.64^{\mathrm{a}}$ \\
\hline & $(6.62)$ & $(8.51)$ & $(7.55)$ & $(3.46)$ & $(6.39)$ \\
\hline \multirow[t]{2}{*}{ AGT-2 } & $15.47^{\mathrm{a}}$ & $17.80^{\mathrm{a}}$ & $16.88^{\mathrm{a}}$ & $12.74^{\mathrm{b}}$ & $15.73^{\mathrm{a}}$ \\
\hline & (7.11) & $(9.35)$ & $(8.44)$ & $(4.86)$ & $(7.35)$ \\
\hline \multirow[t]{2}{*}{ Vaishali } & $14.73^{\mathrm{a}}$ & $18.98^{\mathrm{a}}$ & $20.04^{b}$ & $13.91^{\mathrm{bc}}$ & $16.92^{\mathrm{a}}$ \\
\hline & $(6.47)$ & $(10.58)$ & $(11.75)$ & $(5.78)$ & $(8.47)$ \\
\hline S. Em. \pm & 0.337 & 0.605 & 0.670 & 0.51 & 0.52 \\
\hline $\mathbf{D} \times \mathbf{V}$ & 0.753 & 1.353 & 1.50 & 1.15 & 0.61 \\
\hline V X Y & -- & -- & -- & -- & 0.55 \\
\hline$Y \times D \times V$ & -- & -- & -- & -- & 1.22 \\
\hline C. D. at $5 \%$ & NS & NS & 1.975 & 1.51 & NS \\
\hline $\mathbf{D} \times \mathbf{V}$ & NS & NS & NS & NS & NS \\
\hline V X Y & -- & -- & -- & -- & 1.54 \\
\hline$Y \times D \times V$ & -- & -- & -- & -- & NS \\
\hline C.V. (\%) & 8.68 & 13.08 & 14.72 & 15.94 & 13.41 \\
\hline
\end{tabular}

Note: Figures outside parenthesis are arcsine transformed value and those inside parenthesis are retransformed values, Treatment means with the letter(s) in common are at par by DNMRT at 5\% level of significance. NS: NonSignificant, SMW: Standard Meteorological Week 
Table.4 Impact of sowing period and variety on grain yield of pigeonpea

\begin{tabular}{|c|c|c|c|c|c|}
\hline \multirow[t]{2}{*}{ Treatment } & \multicolumn{5}{|c|}{ Grain yield kg/ha } \\
\hline & 2014-15 & 2015-16 & 2016-17 & 2017-18 & Pooled \\
\hline \multicolumn{6}{|c|}{ Sowing Period (Main Plot Treatment) } \\
\hline $24^{\text {th }}$ SMW ( $2^{\text {nd }}$ week of June $)$ & $1296^{\mathrm{a}}$ & $1408^{\mathrm{a}}$ & $1480^{\mathrm{a}}$ & $1543^{\mathrm{a}}$ & $1432^{\mathrm{a}}$ \\
\hline $26^{\text {th }}$ SMW ( $4^{\text {th }}$ week of June $)$ & $1272^{\mathrm{a}}$ & $1413^{\mathrm{a}}$ & $1365^{\mathrm{ab}}$ & $1353^{\mathrm{a}}$ & $1351^{\mathrm{a}}$ \\
\hline $28^{\text {th }}$ SMW ( $2^{\text {nd }}$ week of July $)$ & $1528^{\mathrm{a}}$ & $1553^{\mathrm{a}}$ & $1212^{\mathrm{bc}}$ & $1312^{\mathrm{a}}$ & $1401^{\mathrm{a}}$ \\
\hline $31^{\text {st }}$ SMW ( $4^{\text {th }}$ week of July $)$ & $1419^{\mathrm{a}}$ & $1667^{\mathrm{a}}$ & $1210^{c}$ & $1207^{\mathrm{a}}$ & $1376^{\mathrm{a}}$ \\
\hline $\begin{array}{l}3^{\text {rd }} \text { SMW ( } 2^{\text {nd }} \text { week of } \\
\text { August })\end{array}$ & $1341^{\mathrm{a}}$ & $1455^{\mathrm{a}}$ & $1196^{\mathrm{c}}$ & $1239^{\mathrm{a}}$ & $1308^{\mathrm{a}}$ \\
\hline$\underset{\text { D. Em. }}{\text { S. }}$ & 58.44 & 77.74 & 47.32 & 72.42 & 32.54 \\
\hline $\mathbf{Y}$ & -- & -- & -- & - & 29.11 \\
\hline D X Y & -- & -- & -- & - & 65.08 \\
\hline $\begin{array}{c}\text { C. D. at } 5 \% \\
\text { D }\end{array}$ & NS & NS & 154.33 & NS & NS \\
\hline $\mathbf{Y}$ & -- & -- & -- & - & 83.05 \\
\hline D x Y & -- & -- & -- & - & 187.48 \\
\hline C.V. (\%) & 12.79 & 15.56 & 10.98 & 16.33 & 14.22 \\
\hline \multicolumn{6}{|l|}{ Variety (Sub Plot Treatment) } \\
\hline BDN-2 & $1368^{\mathrm{b}}$ & $1608^{\mathrm{a}}$ & $1275^{\mathrm{a}}$ & $1352^{\mathrm{a}}$ & $1401^{\mathrm{a}}$ \\
\hline AGT-2 & $1219^{c}$ & $1472^{\mathrm{ab}}$ & $1335^{\mathrm{a}}$ & $1356^{\mathrm{a}}$ & $1345^{\mathrm{a}}$ \\
\hline Vaishali & $1527^{\mathrm{a}}$ & $1417^{\mathrm{b}}$ & $1267^{\mathrm{a}}$ & $1284^{\mathrm{a}}$ & $1374^{\mathrm{a}}$ \\
\hline S. Em. + & 47.90 & 46.45 & 45.56 & 23.21 & 52.76 \\
\hline D x V & 107.098 & 103.88 & 101.87 & 51.90 & 46.99 \\
\hline $\mathbf{Y} \times \mathbf{V}$ & -- & -- & -- & - & 42.03 \\
\hline$Y \times D \times V$ & -- & -- & -- & - & 93.98 \\
\hline C. D. at $5 \%$ & 141.30 & 137.04 & NS & NS & NS \\
\hline D x V & NS & NS & NS & NS & NS \\
\hline $\mathbf{Y} \mathbf{x} \mathbf{V}$ & -- & -- & -- & - & 118.29 \\
\hline$Y \times D \times V$ & -- & -- & -- & - & NS \\
\hline C.V. (\%) & 13.53 & 12.00 & 13.65 & - & 11.85 \\
\hline
\end{tabular}

NS: Non Significant

Results show per cent pod damage pooled over years was significantly different among five periods of sowing. Significantly lowest per cent pod damage was recorded in crop sown during $33^{\text {rd }}$ SMW $(4.31 \%)$ and it was at par with $31^{\text {st }}$ SMW $(4.45 \%), 24^{\text {th }}$ SMW $(5.96 \%)$ and $28^{\text {th }}$ SMW (6.15\%). Significantly highest pod damage was recorded in $26^{\text {th }}$ SMW (6.92\%). Patel et al., (2019) reported that lowest pod damage at green pod stage due to $H$. armigera was observed crop sown in $33^{\text {rd }}$ SMW $(14.93 \%)$ than early sown crop $24^{\text {th }}$ SMW (20.26\%).

\section{Impact of variety}

The data on per cent pod damage by $H$. armigera recorded at the time of the green pod stage of pigeonpea crop are presented in Table 2. During year 2014-15 and 2016- 
17impact of varietyon per cent pod damage was found non-significant.

In the year 2015-16, variety significantly affected pod damage due $H$. armigera. Significantly lowest pod damage was recorded in variety AGT-2 (4.18\%) and highest pod damage recorded in Vaishali $(6.66 \%)$.

During the year 2017-18, significantly lowest per cent grain damage was recorded in AGT-2 $(3.83 \%)$ as compared to Vaishali $(5.43 \%)$ and BDN-2 (6.36\%).

Pooled analysis showed that impact of variety on per cent pod damage green pod stage due to $H$. armigera was non-significant. Further studies are required to confirm above findings.

\section{Impact of sowing period and variety on} pod damage at harvest stage

\section{Impact of sowing period}

The data on the impact of sowing period on pod damage at harvest by $H$. armigera were at green pod stage are given in Table 3. Results show that during the year 2014-15, significantly lowest per cent pod damage was recorded in $33^{\text {rd }}$ SMW $(3.43 \%)$ and it was at par with $31^{\text {st }}$ SMW (4.18\%). The significantly highest pod damage was recorded in $26^{\text {th }}$ SMW (10.60\%).

In the year 2015-16, significantly lowest per cent pod damage was recorded in $33^{\text {rd }}$ SMW $(7.77 \%)$ and it was at par with $31^{\text {st }}$ SMW $(8.10 \%)$ and $28^{\text {th }}$ SMW (8.40\%). Crop sown in $24^{\text {th }}$ SMW recorded significantly highest pod damage (13.50\%). Almost similar trend was observed during the year 2016-17.

In 2017-18, the impact of sowing period on pod damage at harvest was significant. Significantly lowest per cent pod damage was observed in the crop sown in $33^{\text {rd }}$ SMW
(3.97\%) and it was at par with $28^{\text {th }}$ SMW $(4.13 \%)$. Significantly highest per cent pod damage was recorded in crop sown during $24^{\text {th }}$ SMW (5.68\%).

Pooled analysis indicated significantly lowest pod damage was recorded in the crop sown on $33^{\text {rd }}$ SMW (4.79\%) and it was at par with $31^{\text {st }}$ SMW (5.93\%); whereas significantly highest per cent pod damage was observed in crop sown on $24^{\text {th }}$ SMW (9.48). Present finding is in accordance with the earlier work by Patel et al., (2019). They reported lowest per cent pod damage due to $H$. armigera when pigeonpea crop was sown in $33^{\text {rd }}$ SMW $(19.37 \%)$.

\section{Impact of variety}

The data on the impact of variety on pod damage at harvest stage by $H$. armigera are given in Table 3. Results show that during the year 2014-15 and 2015-16 the per cent pod damage was not affected significantly by different varieties.

During the year 2016-17, among three different varieties significantly lowest per cent pod damage was recorded in BDN-2 $(7.55 \%)$ and it was at par with AGT-2 $(8.44 \%)$ whereas significantly highest pod damage observed in Vaishali (11.75\%).

In the year 2017-18, BDN-2 (3.46\%) recorded significantly lowest per cent pod damage as compared to AGT-2 (4.86\%) and Vaishali $(5.78 \%)$.

Pooled analysis indicated that the differences in pod damage among varieties Vaishali, BDN-2 and AGT-2 were non-significant.

Impact of sowing period and variety on grain yield of pigeonpea

\section{Impact of sowing period}

Data on pigeonpea grain yield are given in 
Table 4. During the year 2014-15, 2015-16 and 2017-18 the differences among sowing period for grain yield were non-significant. In the year 2016-17, significantly highest grain was observed in crop sown on $24^{\text {th }}$ SMW $(1480 \mathrm{~kg} / \mathrm{ha})$ and it was at par with $26^{\text {th }} \mathrm{SMW}$ $(1365 \mathrm{~kg} / \mathrm{ha})$. The pooled over years data indicated that sowing period had nonsignificant influence on grain yield of pigeonpea. Late sown and late maturing varieties are vulnerable to the pod fly attack (Sharma et al., 2010). Patel et al. (2019) reported the pod fly attack was lowest in $24^{\text {th }}$ SMW whereas $H$. armigera damage was lowest in $33^{\text {rd }}$ SMW. Hadiya et al., (2020) also reported that the grain damage due to pod fly, M.obtusa was lowest when crop was sown early in $24^{\text {th }}$ SMW as compared to $33^{\text {rd }}$ SMW. Findings of above scientist suggest that early sown crop is more damaged by $H$. armigera, whereas late sown crop is more damaged by $M$. obtusa. This might have caused non-significant impact on grain yield of pigeonpea.

\section{Impact of variety}

Data on pigeonpea grain yield are given in Table 4. Results show that during the year 2014-15, significantly highest grain yield $(1527 \mathrm{~kg} / \mathrm{ha})$ was recorded in variety Vaishali as compared to BDN-2 (1368 kg/ha) and AGT-2 (1219 kg/ha). During the year 201516, BDN-2 (1608 kg/ha) recorded significantly highest grain yield and it was at par with AGT-2 (1472 kg/ha), whereas the lowest grain yield damage found in Vaishali (1417 kg/ha). In the year 2016-17 and 201718 , variety could not influence the grain yield of pigeonpea significantly. Pooled analysis also revealed non-significant influence of variety on grain yield of pigeonpea.

In conclusion pigeonpea crop sown in the $31^{\text {st }}$ SMW ( $4^{\text {th }}$ week of July) to $33^{\text {rd }}$ SMW $\left(2^{\text {nd }}\right.$ week of August) had significantly lower larval population of $H$. armigera, whereas the influence of variety on larval population was non-significant.

Significantly lowest per cent pod damage due to $H$. armigera, recorded at green pod stage, was observed in crop sown in $33^{\text {rd }}$ SMW and it was at par with $31^{\text {st }} \mathrm{SMW}, 28^{\text {th }}$ and $24^{\text {th }}$ SMW. Differences in pod damage among three varieties (Vaishali, BDN-2 and AGT-2) were non-significant.

\section{Acknowledgement}

The authors are thankful to Director of Research and Dean Post Graduate Studies, Anand Agricultural University, Anand for providing all the necessary facilities and encouragement during present investigation. The authors are also thankful to the Unit Head, Agricultural Research Station, Anand Agricultural University, Derol for providing all the necessary facilities during study.

\section{References}

Anonymous, The medium term plan. ICRISAT, Patancheru 502324, Andhra Pradesh, India. 2007; 3: 1-10.

Anonymous. Ministry of Agriculture \& Farmers' Welfare, Govt. of India (ON1953): 2017-18.

Cumming G, Jenkins L. Chickpea. 2011. Effective crop establishment, sowing window, row spacing, seeding depth and rate. Northern Pulse Bulletin, (7): 6.

Davis JC, Lateef. 1975. Insects of pigeonpea and chickpea in India and prospects for control. International Workshop on Grain Legumes, ICRISAT, Hyderabad, India 319-331.

Deka NK, Prasad D, Chand P. 1989. Plant growth; Heliothisincidence and grain yield of chickpea as affected by date of sowing. Journal of Research BirsaAgricultural University, 
Ranchi,1(2):161-168.

Hadiya GD, Patel, SD, Damor, CB, Machhar, RG and Chavadhari, RL. 2020. Impact of Sowing Period and Variety on Pod Fly, Melangromyzaobtusa in Pigeonpea. Journal of Entomology and Zoology Studies, 8(3): 753-757.

PatelHP, Gurjar R, Patel KV and Patel NK. 2019. Impact of sowing periods on incidence of insect pest complex in Pigeon pea. Journal of Entomology and Zoology Studies, 7(2): 1363-1370.

Shanower, T.G. Romeis, J. and Minja, E.M. 1999. Insect pests of pigeonpea and their management. Annual Review of Entomology, 44: 77-96.

Sharma OP, Gopali JB, Yelshetty S, Bambawale OM, Garg DK and Bhosle BB. 2010. Pests of pigeonpea and their management. NCIPM, IARI Campus, Pusa, New Delhi.

Yadava CP, Lal SS, Ahmad R, Sachan JN. 1991. Influence of abiotic factors on relative abundance of pod borers of chickpea (Cicer arietinum). Indian Journal of Agricultural Sciences, 61:512-515.

\section{How to cite this article:}

Hadiya, G. D., S.D. Patel, R. L. Chavadhari, R. G. Machhar and Damor, C. B. 2020. Impact of Sowing Period and Variety on Pod Borer, Helicoverpa armigera Hubner in Pigeonpea. Int.J.Curr.Microbiol.App.Sci. 9(07): 2137-2146. doi: https://doi.org/10.20546/ijcmas.2020.907.249 